\title{
SUMS OF DISTINCT UNIT FRACTIONS
}

PAUL ERDÖS AND SHERMAN STEIN

We shall consider the representation of numbers as the sum of distinct unit fractions; in particular we will answer two questions recently raised by Herbert $S$. Wilf.

A sequence of positive integers $S=\left\{n_{1}, n_{2}, \cdots\right\}$ with $n_{1}<n_{2}<\cdots$ is an $R$-basis if every positive integer is the sum of distinct reciprocals of finitely many integers of $S$. In Research Problem $6[1$, p. 457], Herbert S. Wilf raises several questions about $R$-bases, including: Does an $R$-basis necessarily have a positive density? If $S$ consists of all positive integers and $f(n)$ is the least number required to represent $n$, what, in some average sense, is the growth of $f(n)$ ? These two questions are answered by Theorems 1 and 5 below. Theorem 4 is a "best-possible" strengthening of Theorem 1.

THEOREM 1. There exists a sequence $S$ of density zero such that every positive rational is the sum of a finite number of reciprocals of distinct terms of $S$.

The proof depends on two lemmas.

LEMMA 1. Let $r$ be real, $0<r<1$ and $a_{1}, a_{2}, \ldots$ integers defined inductively by

$$
\begin{aligned}
& a_{1}=\text { smallest integer } n, r-\frac{1}{n} \geqq 0 \\
& a_{2}=\text { smallest integer } n, r-\frac{1}{a_{1}}-\frac{1}{n} \geqq 0, \\
& \vdots \\
& a_{k}=\text { smallest integer } n, r-\frac{1}{a_{1}}-\frac{1}{a_{2}}-\cdots-\frac{1}{a_{k-1}}-\frac{1}{n} \geqq 0 .
\end{aligned}
$$

Then $a_{i+1}>a_{i}\left(a_{i}-1\right)$ for each $i$. Also if $r$ is rational the sequence terminates at some $k$, that is $r=\sum_{i=1}^{k} 1 / a_{i}$.

Lemma 1 is due to Sylvester [2]. It provides a canonical representation for each positive real less than 1 which we will call the Sylvester representation.

Presented to the Society January 29, 1962; received by the editors January 29, 1962. 
LeMma 2. If $r$ is a positive rational and $A$ a positive integer then there exists a finite set of integers $S(r, A)=\left\{n_{1}, n_{2}, \cdots, n_{k}\right\}, n_{1}<n_{2}<\cdots$ $<n_{k}$ such that

$$
\begin{aligned}
r & =\sum_{i=1}^{k} \frac{1}{n_{i}}, \\
n_{1} & \geqq A, \\
n_{i+1}-n_{i} & \geqq A
\end{aligned}
$$

Proof. Since the harmonic series diverges, there is an integer $m$ such that

$$
r-\left(\frac{1}{A}+\frac{1}{2 A}+\cdots+\frac{1}{3 A}+\cdots+\frac{1}{m A}\right)<\frac{1}{(m+1) A} .
$$

Now applying Lemma 1 to

$$
r-\left(\frac{1}{A}+\frac{1}{2 A}+\frac{1}{3 A}+\cdots+\frac{1}{m A}\right)
$$

we conclude that there are integers $m_{1}<m_{2}<\cdots<m_{s}$ such that

$$
r-\left(\frac{1}{A}+\frac{1}{2 A}+\cdots+\frac{1}{m A}\right)=\sum_{i=1}^{i} \frac{1}{m_{i}} .
$$

By our choice of $m$ we see that $m_{1}>(m+1) A$. Moreover Lemma 1 assures us that $m_{i+1}-m_{i}>A$. Then

$$
\left\{A, 2 A, \cdots, m A, m_{1}, m_{2}, \cdots, m_{s}\right\}
$$

serves as $S(r, A)$.

Now the proof of Theorem 1 is immediate. Order the rationals $r_{1}, r_{2}, r_{3}, \cdots$. Let $S_{1}$ be an $S\left(r_{1}, 1\right)$. Let $b_{1}$ be the largest element of $S\left(r_{1}, 1\right)$. Let $S_{2}$ be an $S\left(r_{2}, 2 b_{1}\right)$. Having defined $S_{1}, S_{2}, \cdots, S_{k}$ defines $S_{k+1}$ as follows. Let $b_{k}$ be the largest element of $S_{k}$. Let $S_{k+1}$ be an $S\left(r_{k+1}, 2 b_{k}\right)$.

Then since $S_{k}$ 's are disjoint, there is a monotonically increasing bijection $S:(1,2,3, \cdots) \rightarrow \bigcup_{k=1}^{\infty} S_{k}$ which satisfies the demands of Theorem 1.

In fact $S$ does more than Theorem 1 asserted. It is possible to represent all the positive rationals by sums of reciprocals of terms in the $S$ constructed so that each such reciprocal appears in the representation of precisely one rational. Similar reasoning proves

THEOREM 2. The set of unit fractions $\frac{1}{1}, \frac{1}{2}, \frac{1}{3}, \cdots$ can be partitioned 
into disjoint finite subsets $S_{1}, S_{2}, \ldots$ such that each positive rational is the sum of the elements of precisely one $S_{i}$.

Theorem 2 remains true if the phrase "each positive rational," is replaced by "each positive integer." It would be interesting to know the necessary and sufficient condition that a sequence of rationals $r_{1}, r_{2}, r_{3}, \cdots$ corresponds to the sums of a partition of the set of unit fractions into disjoint finite subsets.

THEOREM 3. If $n_{1}, n_{2}, n_{3}, \cdots$, is a sequence of positive integers with (1) $n_{k+1} \geqq n_{k}\left(n_{k}-1\right)+1$, for $k=1,2,3, \cdots$ and (2) for an infinity of $k, n_{k+1}>n_{k}\left(n_{k}-1\right)+1$ then $\sum_{k=1}^{\infty} 1 / n_{k}$ is irrational. ${ }^{1}$

Proof. Observe first that if $a_{1}, a_{2}, \cdots$ is a sequence of positive integers with $a_{k+1}=a_{k}\left(a_{k}-1\right)+1$ for $k=1,2,3, \cdots$, and $a_{1}>1$, then $\sum_{k=1}^{\infty} 1 / a_{k}=1 /\left(a_{1}-1\right)$. By assumption (2) there is $h$ such that $n_{h}>1$. From the observation we see that for any integer $i$,

$$
\frac{1}{n_{i+1}}<\sum_{k=h}^{\infty} \frac{1}{n_{k}}-\sum_{n=h}^{i} \frac{1}{n_{k}}<\frac{1}{n_{i+1}-1} .
$$

Thus the Sylvester representation of $\sum_{k=h}^{\infty} 1 / n_{k}$ is $1 / n_{h}+1 / n_{h+1}$ $+1 / n_{n+2}+\cdots$. Since the Sylvester representation of $\sum_{n-n}^{\infty} 1 / n_{k}$ has an infinite number of terms, we see by Theorem 1 that $\sum_{n=n}^{\infty} 1 / n_{k}$ is irrational. Hence so is $\sum_{n=1}^{\infty} 1 / n_{k}$ irrational.

We will soon strengthen Theorem 1 by Theorem 4 for which we will need

LEMMA 3. The number of integers in $(x, 2 x)$ all of whose prime factors are $\leqq x^{1 / 2}$ is greater than $x / 10$ for $x>x_{0}$.

PROoF. The number of these integers is at least $x-\sum_{p_{i}}\left(x / p_{i}\right)$, where the summation extends over the primes $x^{1 / 2}<p_{i}<2 x$. From the fact that $\sum_{p<y} 1 / p=\log \log y+c+o(1)$ Lemma 3 easily follows.

THEOREM 4. Let $0<a_{1}<a_{2}<\cdots$ be a sequence $A$ of integers with $\sum_{n=1}^{\infty} 1 / a_{n}=\infty$. Then there exists a sequence $B: b_{1}<b_{2}<\cdots$ of integers satisfying $a_{n}<b_{n}, 1 \leqq n<\infty$, such that every positive rational is the sum of the reciprocals of finitely many distinct b's.

Proof. Set $A(x)=\sum_{a_{i}<x} 1$. We omit from $A$ all the $a_{i}, 2^{k} \leqq a_{i}$ $<2^{k+1}$ for which

$$
A\left(2^{k+1}\right)-A\left(2^{k}\right)<2^{k} / k^{2} .
$$

Thus we obtain a subsequence $A^{\prime}$ of $A, a_{1}^{\prime}<a_{2}^{\prime}<\cdots$. Clearly $\sum_{n=1}^{\infty} 1 / a_{n}^{\prime}=\infty$, since, by (1), the reciprocals of the omitted $a^{\prime}$ s con-

${ }^{1}$ Added in proof. A similar result is to be found in [2]. 
verges.

Set $A^{\prime}(x)=\sum_{a_{i}<x} 1$. Denote by $k_{1}<k_{2}<\cdots$ the integers for which

$$
t_{k_{i}}=A^{\prime}\left(2^{k_{i+1}}\right)-A^{\prime}\left(2^{k_{i}}\right) \geqq 2^{k_{i}} / k_{i}^{2} .
$$

By (2), if $m \neq k_{i}$ then $A^{\prime}\left(2^{m+1}\right)=A^{\prime}\left(2^{m}\right)$.

By Lemma 3 there are at least $\left(t_{k_{i}}\right) / 10$ integers in $\left(2^{k_{i+1}}, 2^{k_{i}+2}\right)$ all of whose prime factors are less than $2^{\left(k_{i}+1\right) / 2}$. Denote such a set of integers by $b_{1}^{(i)}<b_{2}^{(i)}<\cdots<b_{a_{i}}^{(i)}$ where $q_{i}$ is, say, the first integer larger than $t_{k_{i}} / 10$. Clearly

$$
\sum_{r=1}^{a_{i}} 1 / b_{r}^{(i)}>(1 / 40) \sum 1 / a_{j}^{\prime} \quad\left(2^{k_{i}}<a_{j}^{\prime}<2^{k_{i}}+1\right) .
$$

Thus from $\sum 1 / a_{i}^{\prime}=\infty$ we have

$$
\sum_{i=1}^{\infty} \sum_{r=1}^{q_{i}} 1 / b_{r}^{(i)}=\infty
$$

Clearly $b_{q_{i}}^{(i)}<b_{1}^{(i+1)}$; thus all the $b$ 's can be written in an increasing sequence $D: d_{1}<d_{2}<\cdots$.

Now let $u_{1} / v_{1}, u_{2} / v_{2}, \ldots$ be a well-ordering of the positive rationals. Suppose we have already constructed $b_{1}<b_{2}<\cdots<b_{m_{n}}$ so that $a_{i}^{\prime}<b_{i}, 1 \leqq i \leqq m_{n}$ and that $u_{r} / v_{r}, 1 \leqq r<n$, are the sums of reciprocals of distinct $b$ 's. Choose

$$
2^{k_{i}}>\max \left\{v_{n}, b_{m_{n}}, a_{m_{n}}^{\prime}+1\right\}
$$

and let $d_{j_{i+1}}<d_{j_{i+2}}<\cdots$ be the $d$ 's greater than $2^{k_{i+1}}$. By (3) and (4) there is an $s_{i}>j_{i}$ such that

$$
\sum_{r=j_{i}+1}^{n i} 1 / d_{r}<u_{n} / v_{n} \leqq \sum_{r=j_{i+1}}^{1+s_{i}} 1 / d_{r}
$$

By (5)

$$
0<u_{n} / v_{n}-\sum_{j_{i}+1}^{\theta_{i}} 1 / d_{r}=C_{n} / D_{n}<1 / d_{s_{i}} .
$$

Let $x$ be the integer such that $2^{x}<d_{s_{i}} \leqq 2^{x+1}$; then $x=k_{s+1}$ for some $s \geqq i$ (by definition of the $d$ 's). Since, by definition, all the prime factors of $d_{r}, j_{i} \leqq r \leqq q_{i}$ are less than $2^{(x+1) / 2}$ we have

(7) $D_{n} \leqq v_{n}\left[d_{j i+1}, d_{j i+2}, \cdots, d_{s i}\right]<v_{n}\left(2^{x+1}\right)^{2^{(x+1) / 2}}<2^{x}\left(2^{x+1}\right)^{2^{(x+1) / 2}}<2^{2^{2 x / 3}}$ for $x>x_{0}$. 
Now

$$
\frac{C_{n}}{D_{n}}=\frac{1}{y_{1}}+\cdots+\frac{1}{y_{f}}, \quad f<C^{*} \log D_{n}<C 2^{2 x / 3}
$$

with, clearly, $d_{s_{i}}<y_{1}<\cdots<y_{f}$ (by [3]).

Define

$$
\begin{aligned}
b_{m_{n}+t} & =d_{j_{i}+t} & \text { for } t=1, \cdots, s_{i}-j_{i}, \\
b_{m_{n}+s_{i}-j_{i}+t^{\prime}} & =y_{t^{\prime}} & \text { for } 1 \leqq t^{\prime} \leqq f .
\end{aligned}
$$

By (8) the $b$ 's are distinct. Clearly $b_{m_{n}+t}>a_{m_{n}+t}$ for $t=1, \cdots, s_{i}-j_{i}$ since $b_{m_{n}+t}=d_{j_{i}+t}$, and the $d$ 's are greater than the corresponding $a^{\prime \prime}$, which in turn are greater than the $a$ 's. By (8) the $y$ 's do not change the situation. Their number is at most $C 2^{2 x / 3}$. But by (2) there are at least

$$
2^{k_{s}} / k_{s}^{2}>2^{x-1} / x^{2}, \quad x=k_{s}+1
$$

$a_{i}^{\prime}$ 's in $\left(2^{k_{s}}, 2^{k_{s}+1}\right)$ and by definition to more than half of them there does not correspond any $d_{i}$; thus to those $a_{i}^{\prime}$ 's to which no $d$ corresponds we can make correspond the $f<C 2^{2 x / 3} y$ 's since clearly $C 2^{2 x / 3}<2^{x-1} / x^{2}$, if $x>x_{0}$.

The proof is then completed as for Theorem 1 . Note that each $b_{i}$ is used in the representation of only one rational number.

Theorem 4 is a best possible result since if $\sum_{i=1}^{\infty} 1 / a_{h}<\infty$ the conclusion could not possibly hold.

In the next theorem $\boldsymbol{\gamma}$ is Euler's constant.

THEOREM 5. $\lim _{n \rightarrow \infty} f(n) e^{-n}=e^{-\gamma}$.

Proof. Define $g(n)$ by

$$
\frac{1}{1}+\frac{1}{2}+\cdots+\frac{1}{g(n)}<n<\frac{1}{1}+\frac{1}{2}+\cdots+\frac{1}{g(n)}+\frac{1}{g(n)+1} .
$$

Then $n-\sum_{i=1}^{o(n)} 1 / i$ is a rational number less than 1 which we denote $a_{n}$ and which can be expressed in the form

$$
a_{n}=\frac{A}{[1,2, \cdots, g(n)]}
$$

for some integer $A$.

Now, $0<u / v<1$ can be represented as the sum of less than

$$
\frac{c \log v}{\log \log v}
$$


distinct unit fractions [3].

Thus $a_{n}$ is the sum of fewer than

$$
\frac{c \log [1,2, \cdots, g(n)]}{\log \log [1,2, \cdots, g(n)]}
$$

unit fractions (each less than $1 / g(n)$ ). The expression $\log [1,2, \cdots, g(n)]$ is asymptotic to $g(n)[4$, p. 362]. Thus for large $n, a_{n}$ is the sum of fewer than

$$
\frac{c g(n)}{\log g(n)}
$$

distinct unit fractions.

Hence

$$
g(n)<f(n)<g(n)+\frac{c g(n)}{\log g(n)} .
$$

Thus

$$
\lim _{n \rightarrow \infty} f(n) / g(n)=1 \text {. }
$$

From the equation

$$
n=\frac{1}{1}+\frac{1}{2}+\cdots+\frac{1}{g(n)}+a_{n}=\log g(n)+\epsilon_{n}+a_{n}+\gamma
$$

with $\lim _{n \rightarrow \infty} \epsilon_{n}=0$ and $\lim _{n \rightarrow \infty} a_{n}=0$, it follows that $g(n)$ is asymptotic to $e^{n} / e^{\gamma}$.

This proves Theorem 5 .

\section{REFERENCES}

1. Herbert S. Wilf, Reciprocal bases for integers, Bull. Amer. Math. Soc. 67 (1961), 456.

2. J. J. Sylvester, On a point in the theory of vulgar fractions, Amer. J. Math. 3 (1880), 332-335, 388-389.

3. Paul Erdös, The solution in whole numbers of the equation: $1 / x_{1}+1 / x_{2}+1 / x_{3}+$ $\cdots+1 / x_{N}=a / b$, Mat. Lapok 1 (1950), 192-210.

4. G. H. Hardy and E. M. Wright, An introduction to the theory of numbers, 3rd. ed., Oxford Univ. Press, New York, 1954.

TEChNion, Haifa and

University of California at Davis 